Pesq. Vet. Bras. 30(6):484-490, junho 2010

\title{
Anomalias ósseas congênitas em Sotalia guianensis (Mammalia, Cetacea, Delphinidae) da costa centro-norte do estado do Rio de Janeiro ${ }^{1}$
}

\author{
Maíra Laeta ${ }^{2}$, Sheila M.F.M. Souza ${ }^{3}$ e Salvatore Siciliano ${ }^{4}$
}

\begin{abstract}
Laeta M., Souza S.M.F.M. \& Siciliano S. 2010. [Congenital bone defects in Guiana dolphin, Sotalia guianensis (Mammalia, Cetacea, Delphinidae) in the northcentral coast of Rio de Janeiro state, Brazil.] Anomalias ósseas congênitas em Sotalia guianensis (Mammalia, Cetacea, Delphinidae) da costa centro-norte do estado do Rio de Janeiro. Pesquisa Veterinária Brasileira 30(6):484-490. Departamento de Endemias Samuel Pessoa, Escola Nacional de Saúde Pública Sergio Arouca/Fiocruz, Rua Leopoldo Bulhões 1480, Manguinhos, Rio de Janeiro, RJ 21041-210, Brazil. E-mail: mairalaeta @ gmail.com

Carcasses of Guiana dolphins, Sotalia guianensis (van Bénéden, 1864) were recovered from August 20, 2001 to February 13, 2006 along the north-central coast of Rio de Janeiro state. Most of the animals ( $85 \%$ ) were found between Barra de São João $\left(22^{\circ} 35^{\prime}\right.$ S $\left.41^{\circ} 59^{\prime} \mathrm{W}\right)$ and Quissamã ( $\left.22^{\circ} 06^{\prime} S 41^{\circ} 28^{\prime} \mathrm{W}\right)$. We studied the vertebral columns of all the 20 specimens that had more than $60 \%$ of the vertebrae preserved. The series has $75 \%$ of immature animals and all skeletons displayed a minimum of taphonomic changes. Two categories of congenital anomalies were diagnosed, both related to the development of the paraxial mesoderm. The seventh cervical vertebra (C7) was the only affected; the cervical ribs and the cleft neural arches were present in $15(75 \%)$ of the individuals, three (15\%) of which had both anomalies. Nine (45\%) individuals had unilateral or bilateral cervical ribs, and nine (45\%) individuals had cleft neural arches; the contiguous vertebrae were normal in every case. The frequency of these anomalies was higher in that Rio de Janeiro series than in other Sotalia series previously reported in the Brazilian literature for Amazonas, Ceará and Santa Catarina States. The present Sotalia series here described comes from a very limited coastal region probably representing a local population. The cervical ribs are genetically defined and may be concentrated because of a condition of close biological proximity among the animals; the cleft arch could also be determined by environmental factors, to be investigated in the future.
\end{abstract}

INDEX TERMS: Marine mammals, , Guiana dolphins, vertebral column, developmental anomalies, paraxial mesoderm.

RESUMO.- Carcaças de botos-cinza Sotalia guianensis (van Bénéden, 1864) foram recuperadas entre 20 de agosto de 2001 e 13 de fevereiro de 2006, na costa centro-norte fluminense. A maior parte delas (85\%) entre Barra de São

\footnotetext{
${ }^{1}$ Recebido em 9 de dezembro de 2009.

Aceito para publicação em 1 de junho de 2010.

2 Bolsa de pesquisa no Departamento de Endemias Samuel Pessoa, Escola Nacional de Saúde Pública Sergio Arouca/ Fiocruz, Rua Leopoldo Bulhões 1480, sala 606, Rio de Janeiro, RJ 21041-210, Brasil. ${ }^{*}$ Autor para correspondência: mairalaeta@gmail.com

${ }^{3}$ Pesquisadora Titular do Departamento de Endemias Samuel Pessoa, Escola Nacional de Saúde Pública Sergio Arouca/Fiocruz, Rio de Janeiro, RJ.

${ }^{4}$ Pesquisador Visitante (CNPq), Departamento de Endemias Samuel Pessoa, Escola Nacional de Saúde Pública Sérgio Arouca/Fiocruz, Rio de Janeiro, RJ.
}

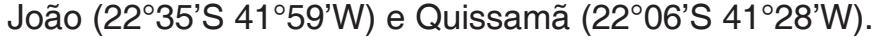
Foram estudadas as colunas vertebrais dos 20 exemplares que apresentavam mais de $60 \%$ das vértebras presentes. A coleção é formada por $75 \%$ de animais imaturos, e todos os esqueletos mostram alterações tafonômicas mínimas. Duas categorias de anomalias congênitas foram diagnosticadas, ambas relacionadas ao desenvolvimento do mesoderma paraxial. A sétima vértebra cervical (C7) foi a única afetada, as costelas cervicais e o não fechamento do arco neural atingiram $15(75 \%)$ dos indivíduos analisados, dos quais três (15\%) apresentam ambas as anomalias. Nove (45\%) indivíduos apresentaram costelas cervicais uni ou bilateral, e nove $(45 \%)$ indivíduos apresentaram não fechamento do arco neural; em todos os 
casos as vértebras contíguas eram normais. A ocorrência de anomalias nesta série do Rio de Janeiro é maior do que as referidas na literatura brasileira para outras séries de Sotalia do Amazonas, Ceará e Santa Catarina. A série de Sotalia descrita vem de uma região do litoral muito limitada e provavelmente representa uma população local. As costelas cervicais são geneticamente determinadas e podem estar concentradas por uma condição de grande proximidade biológica entre os animais; o não fechamento do arco pode ter também um componente ambiental, a ser investigado futuramente.

TERMOS DE INDEXAÇÃO: Mamíferos marinhos, boto-cinza, coluna vertebral, anomalias do desenvolvimento, mesoderma paraxial.

\section{INTRODUÇÃO}

O boto-cinza, Sotalia guianensis (van Bénéden, 1864), está restrito às águas estuarinas e costeiras da porção centro-sul do Oceano Atlântico Oeste, sendo seus limi-

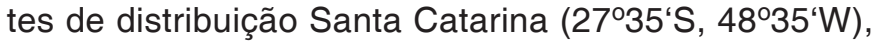
na América do Sul (Simões-Lopes 1988) e Nicarágua $\left(14^{\circ} 35^{\prime} \mathrm{N}, 83^{\circ} 14^{\prime} \mathrm{W}\right)$, na América Central, com ocorrência também em Honduras (Edwards \& Schnell 2001).

A espécie apresenta poucos registros na literatura brasileira sobre anomalias do esqueleto, estando as referências concentradas na coluna vertebral (Furtado \& Simões-Lopes 1999, Fragoso 2001, 2006, Fettuccia et al. 2009). Análises de anomalias ósseas congênitas, ou distúrbios ocorridos durante a morfogênese são recorrentes no que se refere à descrição da costela cervical e do não fechamento do arco neural, na sétima vértebra cervical (C7) (Barnes 1994). Estas lesões origi- nam-se no decurso da segmentação dos somitos, gerada durante ou após processo de neurulação. Ambas estão associadas com as variações do mesoderma paraxial (Barnes 1994). A descrição da ocorrência destas anomalias em Sotalia guianensis, objetiva contribuir para o conhecimento da morfologia e condição genética desta população de mamíferos marinhos, em comparação a outros registros na literatura.

\section{MATERIAL E MÉTODOS}

O material analisado é proveniente da costa centro-norte do estado do Rio de Janeiro, cujos limites geográficos são os municípios de Saquarema (22 $\left.55^{\circ} \mathrm{S} 42^{\circ} 30^{\prime} \mathrm{W}\right)$ e Quissamã ( $\left.22^{\circ} 06^{\prime} S 41^{\circ} 28^{\prime} \mathrm{W}\right)$. Os espécimes estudados estavam esqueletonizados, representando vinte botos-cinza (Sotalia guianensis van Bénéden, 1864) do acervo mantido pelo Grupo de Estudos de Mamíferos Marinhos da Região dos Lagos (GEMM-Lagos), fundado no ano de 2001 e que desde então realiza pesquisas sobre os mamíferos marinhos encalhados na Região dos Lagos, do Estado do Rio de Janeiro. Estes foram recuperados a partir de monitoramentos de praia e registros de encalhes do período entre 30.8.2001 e 13.2.2006, realizados na vigência do Projeto Mamíferos e Quelônios Marinhos da Bacia de Campos. O referido projeto foi coordenado e financiado pela Gerência de Avaliação e Monitoramento Ambiental do Centro de Pesquisas da Petrobras, CENPES/Petrobras (Fig.1, Quadro 1).

Rio de Janeiro, Quissamã: Próximo à Lagoa do Paulista, 2, 31.X.2004, S. Siciliano col. (GEMM 070, GEMM 071). Praia de João Francisco, 1 macho, 10.VIII.2002, S. Siciliano (GEMM 033), 1 macho, 13.II.2006, J.F. Moura (GEMM 096). Flecheiras, 1, 11.XII.2004, S. Siciliano col. (GEMM 076), Flecheiras, 3, 02.VIII.2005, S. Siciliano col. (GEMM 082, GEMM 083, GEMM

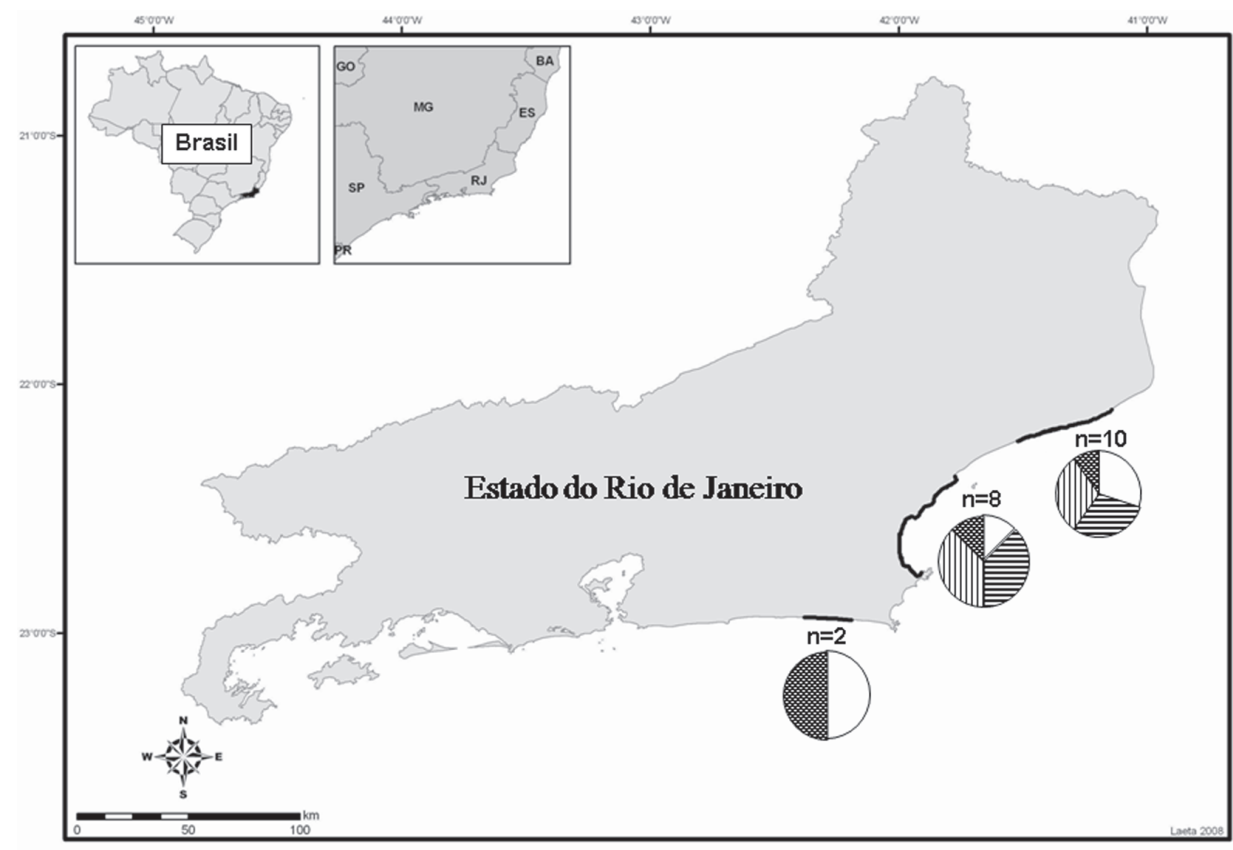

Fig.1. Mapa do estado do Rio de Janeiro destacando os pontos de coleta dos espécimes de Sotalia guianensis provenientes da costa centro-norte fluminense no período de 30.8.2001 a 13.2.2006. Proporção de espécimes com vértebras: $\square$ normais 目 com costela cervical; 而 com não fechamento do arco neural; 圈 com ambas as anomalias. 
Quadro 1. Espécimes de Sotalia guianensis coletados entre 30.8.2001 a 13.2.2006 no centro-norte fluminense pelo GEMM-Lagos, e analisados no presente trabalho, com identificação dos casos de costela cervical uni/bilateral e não fechamento do arco neural

\begin{tabular}{|c|c|c|c|c|c|c|c|}
\hline \multicolumn{2}{|c|}{ № coleção } & \multirow{2}{*}{$\begin{array}{c}\text { Captura/ } \\
\text { encalhe }\end{array}$} & \multirow{2}{*}{$\begin{array}{l}\text { Localidade } \\
\text { São Lázaro, Macaé }\end{array}$} & \multirow{2}{*}{$\begin{array}{c}\text { Sexo } \\
M\end{array}$} & \multirow{2}{*}{$\begin{array}{c}\text { Classe etária } \\
\text { Imaturo }\end{array}$} & \multirow{2}{*}{$\begin{array}{l}\text { Não fechamento } \\
\text { do arco neural } \\
\text { Presente }\end{array}$} & \multirow{2}{*}{$\begin{array}{c}\text { Presença de costela cervical } \\
\text { esquerda(e), direita }(\mathrm{d})\end{array}$} \\
\hline GEMM & 023 & & & & & & \\
\hline GEMM & 031 & $E$ & Praia Seca, Araruama & - & Imaturo & Ausente & - \\
\hline GEMM & 039 & $\mathrm{C}$ & Praião, Barra de São João & $\mathrm{F}$ & Maduro & Ausente & - \\
\hline GEMM & 041 & $\mathrm{E}$ & Praia do Abricó, Rio das Ostras & - & Imaturo & Presente & - \\
\hline GEMM & 047 & $\mathrm{E}$ & Macaé & M & Imaturo & Ausente & e \\
\hline GEMM & 065 & $E$ & Praia da Enseada, Rio das Ostras & - & Maduro & Ausente & $e / d$ \\
\hline GEMM & 068 & $\mathrm{C}$ & Barra de São João & - & Maduro & Presente & - \\
\hline GEMM & 070 & $E$ & Lagoa do Paulista, Quissamã & - & Imaturo & Ausente & $e / d$ \\
\hline GEMM & 071 & $E$ & Lagoa do Paulista, Quissamã & - & Maduro & Ausente & d \\
\hline GEMM & 073 & $E$ & Praia Rasa, Armação dos Búzios & $\mathrm{F}$ & Maduro & Presente & e \\
\hline GEMM & 076 & $E$ & Praia de Flecheiras, Quissamã & - & Imaturo & Presente & - \\
\hline GEMM & 096 & $\mathrm{C}$ & Praia de João Francisco, Quissamã & M & Imaturo & Ausente & e \\
\hline
\end{tabular}

084). Barra do Furado, 1macho, 1fêmea, 05.XI.2003, S. Siciliano col. (GEMM 048, GEMM 049); Macaé: Localidade indeterminada no município de Macaé, 1macho, 18.X.2003, S. Siciliano col. (GEMM 047). Fazenda São Lázaro, 1 macho, 30.VIII.2001, L.B.H.Mendes col. (GEMM 023); Rio das Ostras: Praia do Abricó, 1, 23.XI.2002, S. Siciliano col. (GEMM 041). Praia da Enseada, 1, 02.IX.2004, L.B.H. Mendes (GEMM 065); Casimiro de Abreu: Localidade indeterminada no distrito de Barra de São João, 1, 23.X.2004, V.C. Alves col. (GEMM 068). Praião, 1 fêmea, 09.XI.2002, S. Siciliano col. (GEMM 039), 1 fêmea, 24.IX.2005, S. Siciliano (GEMM 089); Armação dos Búzios: Praia Rasa, 1 fêmea, 18.XI.2004, V.C. Alves col. (GEMM 073); Arraial do Cabo: Figueira, 1macho, 03.IX.2005, S. Siciliano (GEMM 089); Araruama: Praia Seca, 1, 11.VII.2002, F.A. Magalhães (GEMM 031).

Do total de esqueletos recuperados, a maioria (85\%) dos espécimes foi recolhida em um trecho ainda mais restrito, localizado entre Barra de São João (22 $35^{\prime}$ 'S $\left.41^{\circ} 59^{\prime} \mathrm{W}\right)$ e Quissamã (22 $\left.06^{\prime} S 41^{\circ} 28^{\prime} \mathrm{W}\right)$, provavelmente associados à desembocadura do rio São João (Siciliano et al. 2006). A maioria dos espécimes $(95 \%)$ foi coletada no segundo semestre do ano, quando as correntes se dirigem à sudoeste (Bassani et al. 1999, Yoneda 1999), o que parece propiciar o encalhe das carcaças nesta costa. A forma de obtenção do material, captura ou encalhe, não foi considerada neste trabalho, dado o fato de que muitos espécimes já foram encontrados esqueletonizados. Pela sua natureza, esta série não pode ser considerada uma amostra do ponto de vista estatístico, nem uma representação fiel da população viva, já que os espécimes representam a seleção pela mortalidade e pelos fatores que propiciam o seu achado, não se tratando de série obtida de maneira aleatória.

Dentre os espécimes estudados, 15 (75\%) eram imaturos fisicamente, e cinco (25\%) eram maduros. A maturidade física dos exemplares foi determinada de acordo com Perrin (1975) e Fettuccia \& Simões-Lopes (2003). A dificuldade de identificar o sexo dos indivíduos nos esqueletos e carcaças em decomposi- ção, fez com que essa variável não fosse considerada no presente estudo. As alterações tafonômicas foram mínimas, e não prejudicaram o estudo, tendo sido consideradas para sua descrição as alterações propostas por Mello (1999).

Devido à possibilidade de variação no número de componentes nos diferentes segmentos vertebrais de Sotalia guianensis, a fórmula da coluna vertebral usada no presente trabaIho associou a descrição de três trabalhos anteriores (CarvaIho 1963, Da Silva \& Best 1996, Fettuccia \& Simões-Lopes 2004). Foi levada em conta a possibilidade de encontrar-se 7 vértebras cervicais (com o bloco das duas primeiras vértebras, atlas-áxis, fusionado), 10 a 12 vértebras torácicas, 10 a 12 vértebras lombares e 23 a 25 vértebras caudais.

O método empregado foi o estudo morfológico e/ou osteométrico dos ossos, considerando a comparação com o padrão anatômico normal das vértebras, conforme registros da literatura para a espécie (Carvalho 1963, Da Silva \& Best 1994, 1996, Fettuccia \& Simões-Lopes 2004). As variações e anomalias foram consideradas por comparação com humanos, e fundamentadas nas semelhanças da ontogênese dos mamíferos (Barnes 1994, Black \& Scheuer 1997, Almeida 1999, Pough et al. 2003, Souza et al. 2006, Laeta 2007).

Em cada exemplar foram examinadas individualmente todas as vértebras preservadas e desarticuladas, sendo excluídos os exemplares que apresentassem menos de $60 \%$ das vértebras presentes. Todos os exemplares estudados tinham os segmentos, cervical e torácico, preservados. Após exame morfoscópico das vértebras, foram identificados os indivíduos cujas vértebras tinham forma sugestiva de anomalias de formação vertebral, e feita sua osteometria e descrição.

$\mathrm{O}$ diagnóstico de costela cervical em $\mathrm{C} 7$ foi considerado a partir da presença de processos transversos de tamanho alongado, uni ou bilateralmente. Estes processos podiam mostrar estrangulamento ao longo do seu corpo, ou apresentar apenas tamanho proporcionalmente maior em relação ao padrão anatômico predominante para a espécie. Devido à observação de grande variação no tamanho dos processos, a confirmação 
diagnóstica dos casos de costela cervical foi feita a partir de um estudo osteométrico que ajudou a estabelecer os limites entre os ossos normais e os anômalos. A caracterização das costelas cervicais e seu diagnóstico diferencial foram feitos com base em Laeta (2007), tal como descrito a seguir. Para a obtenção das medidas, a vértebra foi posicionada sobre suporte horizontal, com a face cranial para cima. Para referência, foi utilizada uma escala gráfica, e fundo homogêneo e escuro. As fotografias foram feitas com uma câmera digital de 5.1 Megapixels Sony, montada sobre um mini-tripé TR-036L (D-concepts). A distância padrão entre a objetiva e a vértebra foi de $17 \mathrm{~cm}$.

$\mathrm{Na}$ análise osteométrica, proposta e testada pela autora em trabalho anterior (Laeta 2007), buscou-se estabelecer a proporção entre o comprimento do processo transverso e a metade do diâmetro do corpo vertebral. Para este fim duas medidas lineares foram tomadas a partir da projeção do osso na fotografia, e um terceiro valor foi obtido a partir da subtração das primeiras. As medidas foram: (A) distância perpendicular entre a linha média do corpo vertebral e a extremidade do processo transverso; (B) distância perpendicular entre a linha média do corpo vertebral e a paralela que tangencia o contorno lateral da superfície discal. O terceiro valor, representado por $\mathrm{C}$, corresponde, portanto ao comprimento projetado do processo transverso, que foi calculado baseado na subtração A-B.

A partir destas medidas foi feito o cálculo de um índice, em que o valor (B) é multiplicado por 100 e dividido pelo valor $(A)$, conforme a fórmula:

$$
\frac{\mathrm{B} \times 100}{\mathrm{~A}}
$$

Na descrição do não fechamento do arco neural, cuja ocorrência também foi observada em $\mathrm{C}$, foi considerado como critério diagnóstico, a presença de fenda entre as extremidades dos hemi-arcos. A anomalia foi registrada sempre que havia fenda não causada por processo tafonômico ou por fratura.

\section{RESULTADOS}

A costela cervical esteve presente em nove (45\%) indivíduos desta série de $S$. guianensis, podendo ser observada uni ou bilateralmente. E o não-fechamento do arco neural também atingiu nove (45\%) indivíduos. Essas duas condições anômalas coincidiram em três (15\%) espécimes (Quadro 1).

Os gráficos de dispersão mostraram agrupamentos das medidas em dois conjuntos distintos, um formado pelos espécimes normais e outro pelos portadores das costelas cervicais (Fig.2). Dos indivíduos com costelas cervicais, três (33\%) são espécimes maduros e seis (67\%) imaturos fisicamente.

Em relação à simetria, as costelas cervicais foram unilaterais em cinco (56\%) indivíduos, com quatro casos ocorrendo do lado esquerdo e um do lado direito. E nos demais quatro (44\%) indivíduos a ocorrência foi bilateral (Fig.3).

Os valores dos índices ajudaram a estabelecer os limites ou ponto de corte abaixo do qual se considerou a presença de costela cervical. Os valores de índice mais altos que 42 representam os indivíduos normais, e os valores abaixo, representam os espécimes com o processo transverso anômalo ou maior, com exceção de apenas três casos (Quadro 2). Essa relação entre o índice e o tamanho dos processos, é portanto inversamente proporcional.
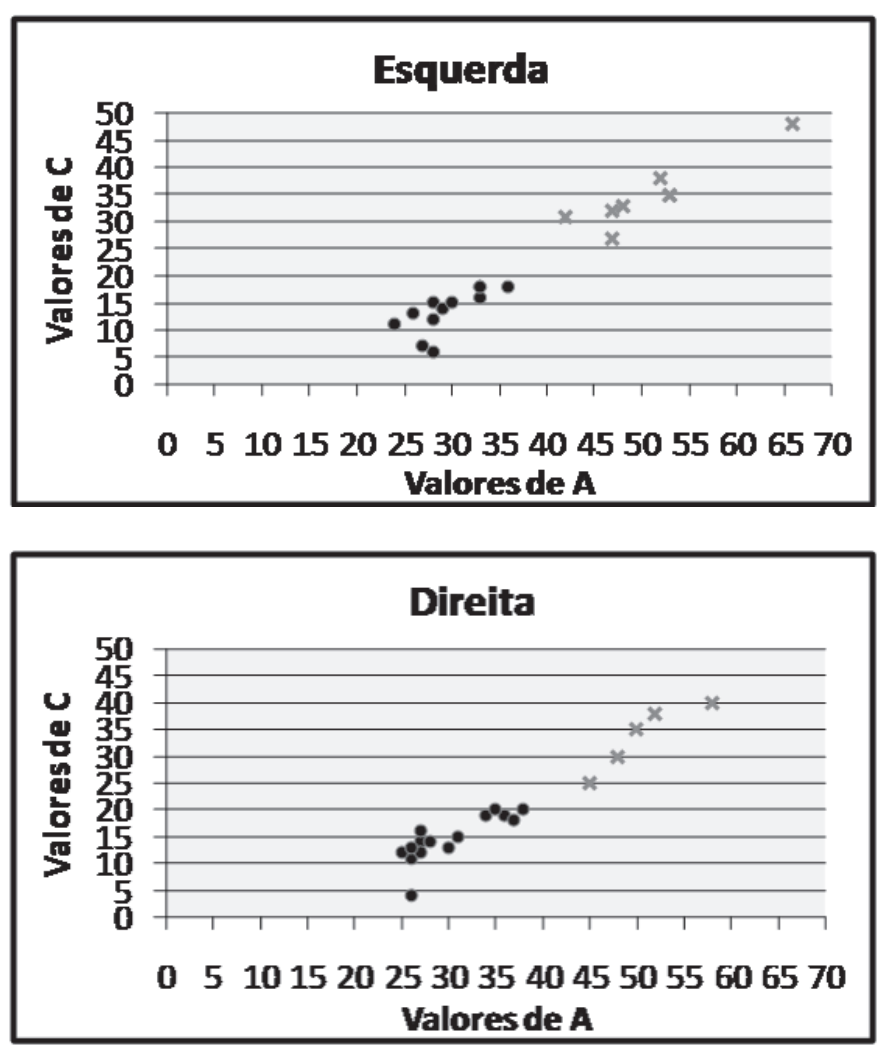

Fig.2. Distribuição das medidas vertebrais $A$ e $C$ nos lados esquerdo e direito das sétimas vértebras cervicais dos espécimes de Sotalia guianensis analisados no presente trabalho. $(\bullet)$ Valores das medidas correspondentes aos exemplares sem costela cervical, e (*) com costela cervical.

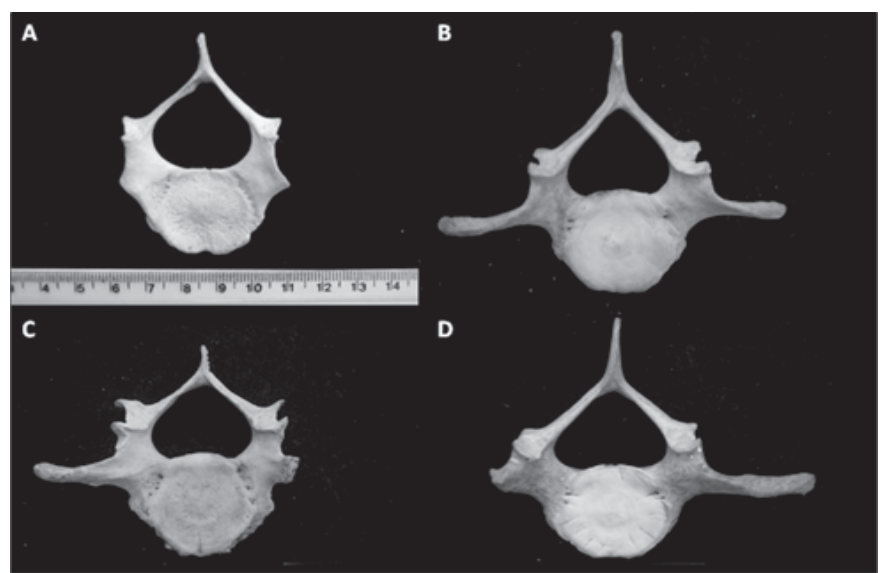

Fig.3. Aspectos da face cranial da sétima vértebra cervical em quatro exemplares estudados: (A) GEMM 049: normal. (B) GEMM 070: costela cervical bilateral. (C) GEMM 071: costela cervical direita. (D) GEMM 096: costela cervical esquerda.

O não fechamento do arco neural esteve presente em nove (45\%) espécimes da coleção estudada. Sendo observadas aberturas na forma de fendas completas no processo neural, que esteve esboçado ou ausente (Fig.4). Em todos os casos as vértebras contíguas eram normais. Dentre 
Quadro 2. Valores das medidas A, B, C e respectivos índices, estimados a partir das vértebras $C 7$ para os Sotalia guianensis analisados no presente trabalho

\begin{tabular}{|c|c|c|c|c|c|c|c|c|}
\hline \multirow{2}{*}{$\begin{array}{l}\text { № de } \\
\text { coleção }\end{array}$} & \multicolumn{4}{|c|}{ Esquerda } & \multicolumn{4}{|c|}{ Direita } \\
\hline & $\mathrm{A}$ & $B$ & C & Índice & A & $B$ & $\mathrm{C}$ & Índice \\
\hline GEMM 023 & 33 & 15 & 18 & 45.4 & 26 & 15 & 11 & 57.7 \\
\hline GEMM 031 & 32 & 17 & 16 & 51.5 & 36 & 17 & 19 & 47.2 \\
\hline GEMM 033 & 28 & 13 & 15 & 46.4 & 27 & 13 & 14 & 48.1 \\
\hline GEMM 039 & 29 & 15 & 14 & 51.7 & 27 & 15 & 12 & 55.5 \\
\hline GEMM 041 & 33 & 15 & 18 & 45.4 & 28 & 14 & 14 & 50 \\
\hline GEMM 047 & 42 & 11 & 31 & 26.2 & 27 & 11 & 16 & 40.7 \\
\hline GEMM 048 & 30 & 15 & 15 & 50 & 34 & 15 & 19 & 44.1 \\
\hline GEMM 049 & 24 & 13 & 11 & 54.2 & 25 & 13 & 12 & 52 \\
\hline GEMM 065 & 53 & 18 & 35 & 34 & 48 & 18 & 30 & 37.5 \\
\hline GEMM 068 & 36 & 18 & 18 & 50 & 30 & 17 & 13 & 56.7 \\
\hline GEMM 070 & 47 & 15 & 32 & 33.3 & 50 & 15 & 35 & 30 \\
\hline GEMM 071 & 27 & 20 & 7 & 74 & 45 & 20 & 25 & 44.4 \\
\hline GEMM 073 & 48 & 15 & 33 & 31.2 & 35 & 15 & 20 & 42.8 \\
\hline GEMM 076 & 28 & 22 & 6 & 78.6 & 26 & 22 & 4 & 84.6 \\
\hline GEMM 082 & 28 & 16 & 12 & 57.1 & 31 & 16 & 15 & 51.6 \\
\hline GEMM 083 & 26 & 13 & 13 & 50 & 26 & 13 & 13 & 50 \\
\hline GEMM 084 & 52 & 14 & 38 & 26.9 & 52 & 14 & 38 & 26.9 \\
\hline GEMM 087 & 53 & 18 & 35 & 34 & 58 & 18 & 40 & 31 \\
\hline GEMM 089 & 47 & 20 & 27 & 42.5 & 37 & 19 & 18 & 51.3 \\
\hline GEMM 096 & 66 & 18 & 48 & 27.3 & 38 & 18 & 20 & 47.4 \\
\hline
\end{tabular}

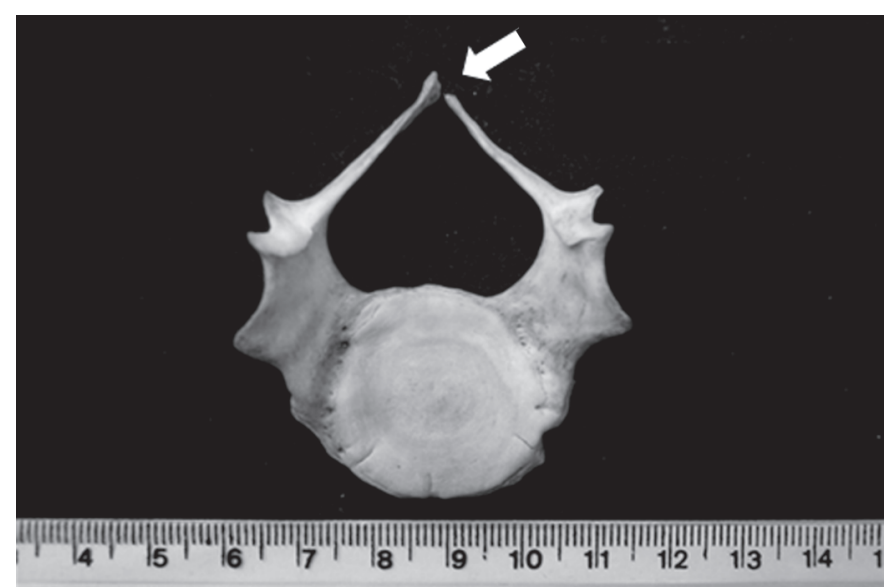

Fig.4. Aspecto da face cranial da sétima vértebra cervical do exemplar GEMM 033 apresentando o não fechamento do arco neural.

os nove indivíduos anômalos, dois (22\%) espécimes eram maduros e sete $(78 \%)$ imaturos fisicamente.

O espécime GEMM 084 apresentou o hemi-arco direito quebrado. Ainda assim o diagnóstico pode ser feito pelo exame do lado esquerdo preservado.

Dentre os portadores do não fechamento do arco neural, três (33\%) espécimes apresentaram também a costela cervical, podendo ser uni ou bilateral.

\section{DISCUSSÃO}

Os espécimes de Sotalia guianensis apresentaram frequência maior do que o esperado para ambas as anomalias congênitas estudadas na coluna vertebral, costela cervical e não fechamento do arco neural, quando comparados os resultados da literatura (Fragoso 2001, 2006, Fettuccia et al. 2009). Estes dados indicam que as causas prováveis dessas anomalias estão presentes de maneira mais expressiva neste grupo.

Em S. guianensis da costa de Santa Catarina esta anoma- lia esteve presente em seis (19\%) dos 32 espécimes analisados e no Ceará esteve presente em dois $(9 \%)$ dos 23 espécimes analisados, com ocorrência geralmente da forma unilateral em ambas as localidades relatadas (Fettuccia et al. 2009). Estes valores mais baixos apontam para o padrão de variabilidade desta característica em tais populações, acentuando o interesse do resultado na série fluminense. O observado neste trabalho difere também pela grande ocorrência bilateral sempre localizada em C7. Relatos isolados desta ocorrência também foram feitos para o gênero, na espécie fluvial, $S$. fluviatilis, onde a prevalência da anomalia foi absoluta, pois dentre os 31 espécimes analisados, 27 (87\%) apresentaram a anomalia, com ocorrência bilateral (Fettuccia et al. 2009).

Segundo Ferigolo (1987) a presença de costelas cervicais nas últimas vértebras da região é uma anomalia que pode ser frequente em algumas espécies de mamíferos, enquanto a ocorrência de costelas não fusionadas às vértebras é um caráter reptiliano. Uma vez que na costa centro-norte fluminense, a frequência foi muito alta, isto contradiz um critério principal citado por Hall $(1984,2005)$ para identificação dos atavismos, para os quais a frequência esperada é sempre muito baixa na população. Deste modo há a possibilidade de que este caráter se explique por polimorfismo.

No caso dos botos-cinza estudados neste trabalho, a ocorrência elevada da anomalia de costelas cervicais na C7, em nove (45\%) espécimes, estaria mais de acordo com a hipótese de origem genética, principalmente se considerarmos que pode tratar-se de um grupo de animais originários de uma mesma região da costa fluminense, talvez pertencente ao mesmo grupo familiar (Di Beneditto \& Ramos 2001, Siciliano et al. 2006). De acordo com Barnes (1994), as costelas cervicais em humanos como em outros mamíferos, são explicadas por uma condição genética ou epigenética, manifestando-se em proporção variável nas diferentes populações.

A hipótese de que estes dados apontem para um grupo de animais com maior frequência de costela cervical na região do centro-norte fluminense, pode significar que as populações originais estão reduzidas, ou apenas indicam que o material estudado representa um grupo muito reduzido local, não representativo da população daquela área de costa. Neste último caso, os animais poderiam estar mostrando nas costelas cenvicais um efeito de deriva genética (Futuyma 1992).

A ocorrência do não fechamento do arco neural em C7 em nove (45\%) espécimes da série atingiu quase metade desta população, indicando que a condição relacionada com a sua expressão está presente nesta população. A porcentagem encontrada pode estar relacionada com fatores ambientais ou genéticos (Barnes 1994, Moalem 2007), já que Fragoso (2001) encontrou apenas um caso semelhante em T1, para o Rio de Janeiro.

No indivíduo vivo a abertura é recoberta por tecido fibroso e a lesão é assintomática, podendo ser relativamente frequente, como em algumas populações humanas, onde geralmente é observada próxima das extremidades da coluna vertebral (Barnes 1994). Tal como ocorre em humanos este é um defeito menor, uma vez que um defeito mais grave provavelmente não seria compatível com a vida dos animais até a idade adulta. 
Em populações humanas a deficiência do ácido fólico ou vitamina $B_{9}$ na dieta das gestantes pode favorecer 0 surgimento deste defeito, cuja manifestação chega a ser endêmica em populações desfavorecidas (Jablonski \& Chaplin 2000). O ácido fólico, um nutriente essencial, é fundamental na biossíntese dos nucleotídeos purina e pirimidina, e posteriormente na biossíntese de ADN (Barnes 1994, Minns 1996, Fleming \& Copp 1998, Jablonski \& Chaplin 2000). Sua ausência ou deficiência ocasionam anomalias fetais, incluindo malformação no esqueleto (Jablonski \& Chaplin 2000, Moalem 2007). Recentemente, em humanos, tem se confirmado que existe uma conexão entre o metabolismo defeituoso do ácido fólico e Defeitos no Tubo Neural (NTD), que compreendem uma família de malformações congênitas. Sua presença pode chegar a impedir $70 \%$ dos defeitos de anencefalia e espinha bífida, normalizando o processo de neurulação em embriões de ratos predispostos (Medical Research Council Vitamin Research Group 1991, Fleming \& Copp 1998). Em cetáceos que vivem em habitat natural, e têm a disponibilidade de capturarem seu alimento, a deficiência de vitamina $\mathrm{B}_{9}$ relacionada à este ciclo metabólico (Fleming \& Copp 1998, Moalem 2007), não parece ser um problema. Por esta razão, não parece provável que uma causa nutricional possa estar relacionada a esta anomalia em animais de vida livre, no entanto cabe uma futura avaliação deste problema.

Estas malformações nos hemi-arcos vertebrais também facilitam, em teoria, a ocorrência de fraturas dos arcos neurais (Souza et al. 2006). O exemplar GEMM 084 apresentou o hemi-arco direito quebrado, neste caso, tal como nos casos clínicos humanos, a fratura perimortem do hemiarco pode ter sido favorecida pela presença da anomalia determinando ponto frágil na estrutura vertebral (Souza 1992).

Malformações na coluna vertebral da família Delphinidae são pouco conhecidas, mas acredita-se que não estejam impossibilitando os espécimes atingirem a vida adulta, pela observação de alguns indivíduos sobrevivendo sob estas condições (Berghan \& Visser 2000, Reiderson 2003, Simões-Lopes 2005).

Malformações congênitas em esqueletos de mamíferos marinhos podem ocorrer inclusive porque, sendo predadores de topo de cadeia, são bioacumuladores, podendo assim sofrer anormalidades durante a morfogênese por efeito de substâncias acumuladas no ambiente e concentradas através de sua dieta (Watson \& Bonde 1986, Weinstein 1995, Winter 1995 apud Berghan \& Visser 2000, Siciliano et al. 2005). Na costa fluminense, Fragoso (2001) relatou que ao longo da distribuição de $S$. guianensis, o local que os esqueletos apresentaram mais malformações foi a área que inclui a baía de Guanabara, o que se associava a contaminação por metais totais e organoclorados em tecidos das carcaças de botos-cinza (Laílson-Brito et al. 2000, 2003, Laílson-Brito et al. 2002 apud Fragoso 2006, Moura et al. 2008). Por esta razão, a presença destas anomalias, ainda que possivelmente relacionadas a uma predisposição genética, devem também ser investigadas quanto aos seus possíveis fatores determinantes ambientais.
Uma vez que são escassos os dados de osteologia em populações vivas desta espécie, torna-se difícil a comparação e a interpretação do significado epidemiológico destes resultados. Por outro lado, o fato de termos, para comparação, outras séries dessa espécie provenientes de regiões distintas e também provenientes de capturas e encalhes, portanto sujeitas a viés semelhante, permite a comparação de seus resultados e a valorização das frequências mais elevadas aqui observadas.

\section{CONSIDERAÇÕES FINAIS}

As anomalias ósseas congênitas de costelas cervicais, uni ou bilaterais, e o não fechamento do arco neural em uma série de Sotalia guianensis da costa centro-norte do Rio de Janeiro, foram identificadas e em seguida descritas e quantificadas.

Os espécimes da costa centro-norte fluminense exibem, até o presente, a maior percentagem de costelas cervicais entre os botos-cinza $S$. guianensis registrados para o litoral brasileiro, entretanto não superiores à percentagem destas nos $S$. fluviatilis.

O não fechamento do arco neural nestes espécimes apresentou porcentagem aparentemente elevada (45\%) embora a literatura não ofereça dados comparativos suficientes tanto para a espécie, quanto para o gênero.

Ainda que o presente estudo tenha permitido constatar esta condição de variação morfológica, outros estudos devem ser feitos para esclarecer a sua etiologia.

Agradecimentos.- Às Dras. Cibele R. Bonvicino, Flavya Almeida-Mendes e Juliana Marigo pelos comentários e sugestões, e ao Grupo de Estudos de Mamíferos Marinhos da Região dos Lagos pelo material prestado. Agradecemos aos revisores pelas críticas na revisão do manuscrito. M. Laeta teve apoio do Programa Institucional de Bolsas de Iniciação Científica (PIBIC) do Conselho Nacional de Desenvolvimento Científico e Tecnológico (CNPq). S. Siciliano é bolsista de produtividade do CNPq. Esta publicação é uma contribuição ao Projeto de Monitoramento de Aves e Mamíferos Marinhos na Bacia da Campos/CENPES/ Petrobras e do Departamento de Endemias Samuel Pessoa/DENSP, Escola Nacional de Saúde Pública Sergio Arouca/ENSP, Fiocruz.

\section{REFERÊNCIAS}

Almeida J.M. 1999. Embriologia Veterinária Comparada. Guanabara Koogan, Rio de Janeiro. 176p.

Barnes E. 1994. Developmental Defects of the Axial Skeleton in Paleopathology. University Press of Colorado, Niwot. 360p.

Bassani C., Bonecker A.C.T., Bonecker S.L.C., Nogueira C.R., Reis J.M.L. dos \& Nascimento L.R. 1999. Plâncton do litoral norte do Estado do Rio de Janeiro (2100' a $23^{\circ} 30^{\prime} S$ ): análise e síntese do conhecimento, p.99-120. In: Silva S.H.G. \& Lavrado H.P. (Eds), Ecologia dos Ambientes Costeiros do Estado do Rio de Janeiro. Vol.2. Série Oecologia Brasiliensis, Rio de Janeiro.

Berghan J. \& Visser I.N. 2000. Vertebral column malformations in New Zeland delphinids with a review of cases worldwide. Aq. Mamm. 26:17-25.

Black S. \& Scheuer L. 1997. The Ontogenetic development of the cervical rib. Int. J. Osteoarchaeol. 7:2-10.

Carvalho C.T. 1963. Sobre um boto comum no litoral do Brasil (Cetacea, Delphinidae). Revta Bras. Biol. 23:266-276.

Da Silva V.M.F. \& Best R.C. 1994. Tucuxi Sotalia fluviatilis (Gervais, 1853). In: Ridgway S.H. \& Harrison R.J. (Eds), Handbook of Marine Mammals. Academic Press, London. 416p.

Da Silva V.M.F. \& Best R.C. 1996. Sotalia fluviatilis. Mamm. Spec. 527:1-7. 
Di Beneditto A.P.M. \& Ramos R.M.A. 2001. Os cetáceos da Bacia de Campos. Ciência Hoje 2:66-69.

Edwards H.H. \& Schnell G.D. 2001. Status and ecology of Sotalia fluviatilis in the Cayos Miskito Reserve, Nicaragua. Mar. Mamm. Sci. 17:445-472.

Ferigolo J. 1987. Anatomia comparada, paleontologia e paleopatologia de vertebrados. Paula-Coutiana 1:105-127.

Fettuccia D.C., Da Silva V.M.F. \& Simões-Lopes P.C. 2009. Nonmetric characters in two species of Sotalia (Gray, 1866) (Cetacea, Delphinidae). Braz. J. Biology 69(3):907-917.

Fettuccia D.C. \& Simões-Lopes P.C. 2003. Variações ontogenéticas na coluna vertebral do boto-cinza (Sotalia guianensis). $2^{\circ}$ Congresso Brasileiro de Mastozoologia, Belo Horizonte, MG, p.58. (Resumo)

Fettuccia D.C. \& Simões-Lopes P.C. 2004. Morfologia da coluna vertebral do boto-cinza, Sotalia guianensis (Cetacea, Delphinidae). Biotemas 17:125-148.

Fleming A. \& Copp A.J. 1998. Embryonic folate metabolism and mouse neural tube defects. Science 280:2107-2109.

Fragoso A.B.L. 2001. alterações morfológicas e patológicas em esqueletos de Boto-cinza Sotalia fluviatilis (Gervais, 1853) do Litoral do Estado do Rio de Janeiro. Dissertação de Mestrado em Ciências Biológicas, Museu Nacional, Universidade Federal do Rio de Janeiro, Rio de Janeiro, RJ. 136p.

Fragoso A.B.L. 2006. Alterações morfológicas e patológicas em esqueletos e nadadeiras peitorais de Boto-cinza, Sotalia guianensis (van Bénéden, 1864) do Litoral Brasileiro. Tese de Doutorado em Ciências Biológicas, Museu Nacional, Universidade Federal do Rio de Janeiro, Rio de Janeiro, RJ. 130p.

Furtado M.H.B.C. \& Simões-Lopes P.C. 1999. Alterações senil-degenerativas e variações anatômicas na coluna vertebral de pequenos cetáceos. Biotemas 12:133-144.

Futuyma D.J. 1992. Biologia Evolutiva. Sociedade Brasileira de Genética/CNPq, Ribeirão Preto. 646p.

Hall B.K. 1984. Developmental mechanisms underlying the formation of atavisms. Biol. Rev. Camb. Phil. Soc. 78:409-433.

Hall B.K. 2005. Bones and cartilage: Developmental and evolutionary skeletal biology. Elsevier Academic Press, San Diego. 760p.

Jablonski N.G. \& Chaplin G. 2000. The evolution of human skin coloration. J. Hum. Evol. 39:57-106.

Laeta M. 2007. Anomalias ósseas congênitas em Sotalia guianensis van Bénéden, 1864 (Cetacea, Delphinidae) da costa centro-norte do estado do Rio de Janeiro, Brasil. Monografia em Ciências Biológicas, Instituto de Biologia, Universidade Federal Fluminense, Niterói, RJ. 70p.

Laílson-Brito J.Jr, Azeredo M.A.A., Saldanha M.F.C., Fernandez M.A., Ramos R.M.A., Di Beneditto A.P.M. \& Herms F.W. 2000. Estudo ecotoxicológico das concentrações de metais-traço em tecidos de cetáceos do Brasil. 9a Reunión de Trabajo de Especialistas en Mamíferos Acuáticos de América Del Sur, Buenos Aires, Argentina, p.67. (Resumo)

Laílson-Brito J.Jr, Meire R.O., Silva C.E.A., Massena E.P., Azeredo A., Torres J.P.M. \& Malm O. 2003. DDT and PCB in blubber of killer whale, Orcinus orca, and marine tucuxi dolphin, Sotalia fluviatilis, from Rio de Janeiro state, Brazil: Preliminary results. Organohalogen Compd. 62:364-366.

Medical Research Council Vitamin Research Group 1991. Prevention of neural tube defects: results of the Medical Research Council vitamin study. Lancet 338:131-137.

Mello M.G.S. 1999. Sistematização de critérios para diagnóstico diferencial entre paleopatologias e sinais de alterações análogas: fundamentos teórico-metodológicos. Tese de Doutorado em Saúde Pública, Escola Nacional de Saúde Pública Sérgio Arouca/Fiocruz, RJ. 211p.

Minns R. 1996. Folic acid and neural tube defects. Spinal Cord 34: 460-465.

Moalem S. 2007. A sobrevivência dos mais doentes: um estudo radical das doenças como fator de sobrevivência. Elsevier Academic Press, Rio de Janeiro. 242p.

Moura J.F., Siciliano S., Sarcinelli P.N. \& Hacon S. 2008. Several organochlorine pesticides in the milk of a marine tucuxi dolphin incidentally captured with its calf in Barra de São João, east coast of Rio de Janeiro state, Brazil. Marine Biodiversity Records, Plymouth. Disponível em <http://www.mba.ac.uk/jmba/pdf/6201.pdf > Acessado em 30 out. 2008.

Perrin W.F. 1975. Variation of spotted and spinner porpoises (genus Stenella) in the Eastern Pacific and Hawaii. Bull. Scripps Inst. Oceanogr. 21:1-206.

Pough F.H., Janis C.M. \& Heiser J.B. 2003. A Vida dos Vertebrados. Atheneu, São Paulo. 596p.

Reiderson T.H. 2003. Zoo and Wild Animal Medicine. W.B. Saunders, St Louis, p.442-459.

Siciliano S., Alves V.C. \& Hacon S. 2005. Aves e mamíferos marinhos como sentinelas ecológicas de saúde ambiental: uma revisão do conhecimento brasileiro. Cad. Saúde Col. 13:927-946.

Siciliano S., Moreno I.B., Silva E.D. \& Alves V.C. 2006. Baleias, botos e golfinhos na Bacia de Campos. Série Guias de Campo: Fauna Marinha da Bacia de Campos. São Miguel, Caxias do Sul. 100p.

Simões-Lopes P.C. 1988. Ocorrência de uma população de Sotalia fluviatilis Gervais, 1853 (Cetacea, Delphinidae) no limite sul de sua distribuição, Santa Catarina, Brasil. Biotemas 1:57-62.

Simões-Lopes P.C. 2005. O Luar do Delfim: a maravilhosa aventura da história natural. Letradágua, Joinville. 304p.

Souza S.M.F.M. 1992. Traumatismos vertebrais como indicadores de atividade física na população da Furna do Estrago, Pernambuco, Brasil, p.123-140. In: Araújo A. \& Ferreira L.F. (Eds), Paleopatologia e Paleoepidemiologia: estudos multidisciplinares. Panorama ENSP, Rio de Janeiro. 237p.

Souza S.M.F.M., Laeta M. \& Siciliano S. 2006. Lesões ósseas em colunas vertebrais de golfinhos do gênero Sotalia provenientes do litoral norte do estado do Rio de Janeiro, Brasil. Workshop on Research and Conservation of the Genus Sotalia, Armação dos Búzios, RJ, p.32. (Resumo)

Watson A.G. \& Bonde R.K. 1986. Congenital malformations of the flipper in three West Indian manatees, Trichechus manatus, and a proposed mechanism for development of ectrodactyly and cleft hand in mammals. Clin. Orthop. Rel. Res. 202:294-301.

Weinstein S.L. 1995. Deformities of the spine, p.195-230. In: Weinstein J.N., Rydevik B.L. \& Sonntag V.K.H. (Eds), Essentials of the Spine. Raven Press, New York.

Yoneda N.T. 1999. Área temática: plâncton. In: Workshop para Avaliação e Ações Prioritárias para a Conservação da Biodiversidade da Zona Costeira e Marinha do Paraná. Disponível em <http://www.anp. gov.br/brnd/round6/guias/PERFURACAO/PERFURACAO_R6/refere/ pl\%E2ncton.pdf> Acesso em 10 jun. 2008. 